Research Article

\title{
An upper bound for the Laplacian index of a signed graph
}

\author{
Farzaneh Ramezani ${ }^{1}$, Zoran Stanić $^{2, *}$ \\ ${ }^{1}$ Faculty of Mathematics, K.N. Toosi University of Technology, P.O. Box 16315-1618, Tehran, Iran \\ ${ }^{2}$ Faculty of Mathematics, University of Belgrade, Studentski trg 16, 11000 Belgrade, Serbia
}

(Received: 25 November 2020. Accepted: 16 February 2021. Published online: 20 February 2021.)

(c) 2021 the authors. This is an open access article under the CC BY (International 4.0) license (www.creativecommons.org/licenses/by/4.0/)

\begin{abstract}
We prove that

$$
\mu_{1} \leq \max \left\{\sqrt{2\left(d_{i}^{2}+d_{i} m_{i}-2 T_{i}^{+}\right)}: 1 \leq i \leq n\right\},
$$

where $\mu_{1}$ is the Laplacian index of a signed graph $\dot{G}$ with $n$ vertices and, for a vertex $i$, the symbols $d_{i}, m_{i}$ and $T_{i}^{+}$denote its degree, average 2-degree and the number of positive triangles containing $i$, respectively. We also show that equality holds if and only if $\dot{G}$ is switching equivalent to a regular signed graph with all edges being negative. Apart from this result, we derive some other upper bounds for $\mu_{1}$, make some comparisons and conclude by finding a lower bound for the same eigenvalue.
\end{abstract}

Keywords: signed graph; largest eigenvalue; vertex degree; regular signed graph.

2020 Mathematics Subject Classification: 05C22, 05C50.

\section{Introduction}

A signed graph $\dot{G}$ is a pair $(G, \sigma)$, where $G=(V, E)$ is an unsigned graph, called the underlying graph, and $\sigma: E \longrightarrow$ $\{+1,-1\}$ is the sign function. The edge set of a signed graph is composed of subsets of positive and negative edges. We denote the number of vertices of a signed graph by $n$.

The $n \times n$ adjacency matrix $A_{\dot{G}}$ of $\dot{G}$ is obtained from the standard $(0,1)$-adjacency matrix of $G$ by reversing the sign of all 1 s which correspond to negative edges. If $D_{\dot{G}}$ is the diagonal matrix of vertex degrees, then the Laplacian matrix of $\dot{G}$ is defined as $L_{\dot{G}}=D_{\dot{G}}-A_{\dot{G}}$. The eigenvalues of $L_{\dot{G}}$ are real and non-negative, and they form the Laplacian spectrum of $\dot{G}$. The largest eigenvalue of $L_{\dot{G}}$ is called the Laplacian index and denoted by $\mu_{1}$.

Spectra of standard matrices associated with signed graphs have received a great deal of attention in the recent past. It occurs that, as in the particular case of graphs, the largest eigenvalue is the most investigated one. It gives information about the structure and has applications in various fields. Some details can be found in $[5,7,8]$. For some recently obtained upper bounds, we refer the reader to [3,4].

Section 2 contains some definitions, additional terminology and notation. Our contribution is reported in Section 3. The main result is the sharp upper bound given at the beginning of that section. It is followed by some other bounds and their comparisons.

\section{Preparatory}

For $U$ a subset of the vertex set, let $\dot{G}^{U}$ be the signed graph obtained from $\dot{G}$ by reversing the sign of each edge between a vertex in $U$ and a vertex in $V(\dot{G}) \backslash U$. The signed graph $\dot{G}^{U}$ is said to be switching equivalent to $\dot{G}$. The switching equivalence is an equivalence relation and switching equivalent signed graphs share the same Laplacian spectrum.

We denote by $d_{i}$ the degree of a vertex $i \in V(\dot{G})$. We also write $d_{i}^{+}$and $d_{i}^{-}$for the positive and negative vertex degree (i.e., the number of positive and negative edges incident with $i$ ). If the vertices $i$ and $j$ are adjacent, then we write $i \sim j$. The existence of a positive (resp. negative) edge between these vertices is designated by $i \stackrel{\sim}{\sim} j\left(i \bar{\sim} j\right.$ ). For $d_{i}>0$, we denote

$$
m_{i}^{+}=\frac{1}{d_{i}}\left(\sum_{\substack{j \pm i \\ j \sim i}} d_{j}^{+}+\sum_{j \bar{i}} d_{j}^{-}\right), m_{i}^{-}=\frac{1}{d_{i}}\left(\sum_{\substack{ \pm \sim i \\ j \sim i}} d_{j}^{-}+\sum_{j \bar{\sim} i} d_{j}^{+}\right)
$$

\footnotetext{
*Corresponding author (zstanic@math.rs).
} 
and $m_{i}=m_{i}^{+}+m_{i}^{-}$; the last parameter is known as the average 2-degree, that is the average degree of the neighbours of $i$. For $d_{i}=0$, we define all these parameters to be zero.

For the vertices $i$ and $j$, we use $c_{i j}$ to denote the number of their common neighbours (i.e., joined to both of them by any edge), $c_{i j}^{+}$for the number of common neighbours joined to $i$ by a positive edge and to $j$ by any edge, and $c_{i j}^{++}$for the number of common neighbours that are joined to both of them by a positive edge. We also use the similar notation for all the remaining possibilities.

A cycle in $\dot{G}$ is said to be positive if the number of its negative edges is even. Otherwise, it is called negative. In particular, the number of positive triangles that contain a fixed vertex $i$ is denoted by $T_{i}^{+}$.

If $\mathbf{x}=\left(x_{1}, x_{2}, \ldots, x_{n}\right)^{\top}$ is an eigenvector associated with the Laplacian eigenvalue $\mu$, then from $L_{\dot{G}} \mathbf{x}=\mu \mathbf{x}$, we get the eigenvalue equation (for $\mu$ ):

$$
\mu x_{i}=d_{i} x_{i}-\sum_{u \sim i} \sigma(u i) x_{u}
$$

Since every graph can be interpreted as a signed graph with all the edges being positive, all notions defined in this section are automatically defined for graphs. Observe that the standard Laplacian matrix of a graph $G$ coincides with the Laplacian matrix of the same graph where it is interpreted as a signed graph. The standard signless Laplacian matrix of $G$ coincides with the Laplacian matrix of the signed graph obtained by reversing the sign of all the edges of $G$.

\section{Results}

Our main contribution reads as follows.

Theorem 3.1. For the Laplacian index $\mu_{1}$ of a signed graph $\dot{G}$ with $n$ vertices,

$$
\mu_{1} \leq \max \left\{\sqrt{2\left(d_{i}^{2}+d_{i} m_{i}-2 T_{i}^{+}\right)}: 1 \leq i \leq n\right\},
$$

where, for a vertex $i, d_{i}, m_{i}$ and $T_{i}^{+}$denote its degree, average 2-degree and the number of positive triangles containing $i$, respectively.

Equality holds if and only if $\dot{G}$ is switching equivalent to a regular signed graph with all edges being negative.

Proof. Let $\mathbf{x}$ be an eigenvector associated with $\mu_{1}$, and let $x_{i}$ be the maximum absolute value of the coordinates of $\mathbf{x}$. Without loss of generality, we may assume that $x_{i}=1$. Using the eigenvalue equation (1), we get

$$
\mu_{1}=d_{i}-\sum_{u \sim i} \sigma(u i) x_{u}=d_{i}-\sum_{\substack{u \pm i \\ u \sim j}} x_{u}-\sum_{\substack{u \neq i \\ u \neq j \\ u \sim j}} x_{u}-\sum_{\substack{u \pm i \\ u \sim j \\ u \sim j}} x_{u}+\sum_{\substack{u \bar{\sim} \\ u \nsim j}} x_{u}+\sum_{\substack{u \sim i \\ u \neq j}} x_{u}+\sum_{\substack{u \sim i \\ u \sim j \\ u \sim j}} x_{u} .
$$

Similarly, for an arbitrary neighbour of $i$, say $j$, we have

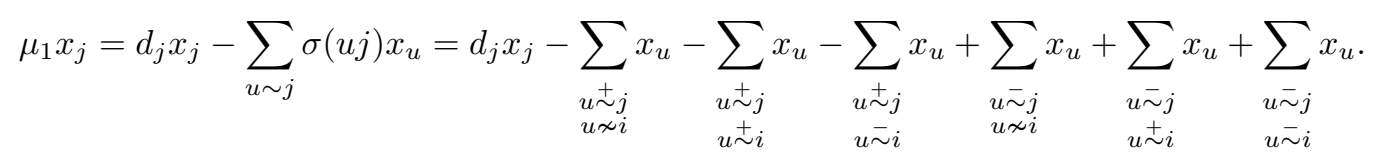

By adding (4) to (3), we get

$$
\mu_{1}\left(1+x_{j}\right)=d_{i}+d_{j} x_{j}-\sum_{\substack{u \sim i \\ u \nsim j}} x_{u}-2 \sum_{\substack{u \sim i \\ u \sim j}} x_{u}+\sum_{\substack{u \sim i \\ u \nsim j}} x_{u}+2 \sum_{\substack{u \sim i \\ u \sim j}} x_{u}-\sum_{\substack{u \sim j \\ u \nsim j}} x_{u}+\sum_{\substack{u \sim j \\ u \nsim j \\ u \nsim i}} x_{u} .
$$

This implies

$$
\begin{aligned}
\mu_{1}\left(1+x_{j}\right) & \leq d_{i}+d_{j}+d_{i}^{+}-c_{i j}^{+}+2 c_{i j}^{++}+d_{i}^{-}-c_{i j}^{-}+2 c_{i j}^{--}+d_{j}^{+}-c_{j i}^{+}+d_{j}^{-}-c_{j i}^{-} \\
& =2 d_{i}+2 d_{j}-2 c_{i j}+2 c_{i j}^{++}+2 c_{i j}^{--}=2 d_{i}+2 d_{j}-2 c_{i j}^{+-}-2 c_{i j}^{-+} .
\end{aligned}
$$

Taking the summation over all $j$ such that $j \bar{\sim} i$, we get

$$
\mu_{1}\left(d_{i}^{-}+\sum_{j \overline{ }} x_{j}\right) \leq 2 d_{i} d_{i}^{-}+2 \sum_{j \bar{i}} d_{j}-2 \sum_{j \bar{i}} T_{i j}^{+} .
$$

Acting in a similar way, by subtracting (4) from (3), we get

$$
\mu_{1}\left(1-x_{j}\right)=d_{i}-d_{j} x_{j}-\sum_{\substack{u \neq i \\ u \nsim j}} x_{u}-2 \sum_{\substack{u \neq i \\ u \sim j \\ u \sim j}} x_{u}+\sum_{\substack{u \sim i \\ u \nsim j}} x_{u}+2 \sum_{\substack{u \sim i \\ u \sim j}} x_{u}+\sum_{\substack{u \neq j \\ u \nsim i}} x_{u}-\sum_{\substack{u \sim j \\ u \sim j \\ u \nsim i}} x_{u},
$$


which gives

$$
\begin{aligned}
\mu_{1}\left(1-x_{j}\right) & \leq d_{i}+d_{j}+d_{i}^{+}-c_{i j}^{+}+2 c_{i j}^{+-}+d_{i}^{-}-c_{i j}^{-}+2 c_{i j}^{-+}+d_{j}^{+}-c_{j i}^{+}+d_{j}^{-}-c_{j i}^{-} \\
& =2 d_{i}+2 d_{j}-2 c_{i j}+2 c_{i j}^{+-}+2 c_{i j}^{-+}=2 d_{i}+2 d_{j}-2 c_{i j}^{++}-2 c_{i j}^{--} .
\end{aligned}
$$

Taking the summation over all $j$ such that $j \stackrel{\perp}{\sim} i$, we get

$$
\mu_{1}\left(d_{i}^{+}-\sum_{\substack{j^{+} i \\ j \sim i}} x_{j}\right) \leq 2 d_{i} d_{i}^{+}+2 \sum_{\substack{j^{+} i \\ \sim}} d_{j}-2 \sum_{\substack{j^{+} i \\ \text { i }}} T_{i j}^{+} .
$$

By taking the sum of (6) and (8), we get

$$
\mu_{1}\left(d_{i}-\sum_{j \sim i} \sigma(i j) x_{j}\right) \leq 2 d_{i}^{2}+2 \sum_{j \sim i} d_{j}-2 \sum_{j \sim i} T_{i j}^{+} .
$$

By using the eigenvalue equation once again, we arrive at

$$
\mu_{1}^{2} \leq 2 d_{i}^{2}+2 \sum_{j \sim i} d_{j}-2 \sum_{j \sim i} T_{i j}^{+}
$$

i.e.,

$$
\mu_{1} \leq \sqrt{2 d_{i}^{2}+2 d_{i} m_{i}-4 T_{i}^{+}}
$$

Finally, taking the maximum over all vertices, we obtain (2).

If equality in (2) holds, then we have equality in (5) and (7). Considering both, we get

$$
\begin{aligned}
& x_{j}=1 \quad \text { if } i \bar{\sim} j, \quad \text { and } \quad x_{u}=1 \quad \text { if } u \bar{\sim} j, \\
& x_{j}=-1 \quad \text { if } i \stackrel{\sim}{\sim} j \quad \text { and } \quad x_{u}=-1 \quad \text { if } u \stackrel{ \pm}{\sim} j .
\end{aligned}
$$

In other words, the coordinates of $\mathbf{x}$ are equal in absolute value and if two vertices are adjacent by a negative edge then the corresponding coordinates are equal, while if two vertices are adjacent by a positive edge then the corresponding coordinates differ in sign. Since $x_{i}=1$, it follows by (9) that every pair of vertices adjacent by a negative edge correspond to 1 in $\mathbf{x}$. By making a switch with respect to all the vertices which correspond to -1 in $\mathbf{x}$, we arrive at the signed graph, say $\dot{H}$, with all the edges being negative. Simultaneously, $\mathbf{x}$ is transformed to the all-1 eigenvector $\mathbf{j}$ which corresponds to the Laplacian index of $\dot{H}$. Since $L_{\dot{H}}$ is, in fact, the signless Laplacian matrix of the underlying graph $H$, we conclude that $\dot{H}$ must be regular (as $\mathbf{j}$ corresponds to its largest eigenvalue - see [6, p. 23]).

Conversely, if $\dot{G}$ is switching equivalent to the regular signed graph (of vertex degree $r$ ) with all the edges being negative, then (2) reduces to $\mu_{1} \leq \sqrt{4 r^{2}}=2 r$ (since $T_{i}^{+}=0$, for all $i$ ). Moreover, the signless Laplacian index of a regular graph is equal to $2 r$, and we are done.

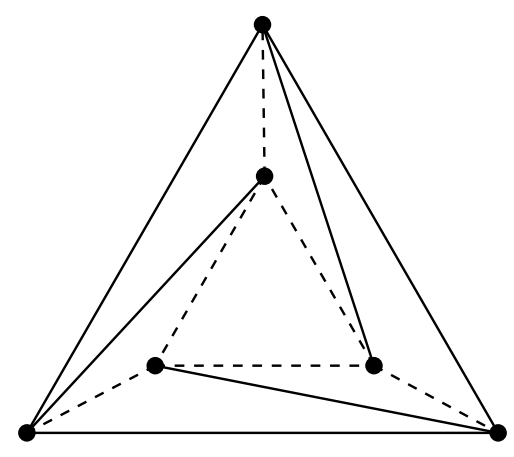

Figure 1: The signed graph for Example 3.1. Negative edges are dashed.

A similar proof technique in case of unsigned graphs can be found in Das's [1]. Our proof differs since it concerns signed graphs, has a more complicated computation which in particular contains a part that results in inequality (6) and, possibly the most important, resolves the case of equality. Here is an example.

Example 3.1. Consider the signed graph illustrated in Figure 1. Its Laplacian index is equal to 6. Since every vertex belongs to exactly two positive triangles, the upper bound of (2) is equal to $2 \sqrt{14} \approx 7.48$. By taking all the edges to be positive, we obtain the signed graph whose Laplacian index is also equal to 6, but now the upper bound gives a better estimate since every vertex belongs to exactly 4 positive triangles; it is $4 \sqrt{3} \approx 6.93$. Of course, by taking all the edges to be negative, we get the equality in (2); both sides are equal to 8. 
Consider now the particular case of graphs. For the Laplacian index, the bound (2) remains unchanged, since every triangle of a graph is positive.

For the signeless Laplacian index $\kappa_{1}$, the upper bound (2) reduces to

$$
\kappa_{1} \leq \max \left\{\sqrt{2 d_{i}\left(d_{i}+m_{i}\right)}: 1 \leq i \leq n\right\},
$$

since the corresponding graph is interpreted as a signed graph with all edges being negative. The bound (10) is known and can be found in [5, p. 207]. The same bound is derived for the Laplacian index in [2]; evidently, our bound (2) (used for the Laplacian index of a graph) improves this result.

From (1), we get $\kappa x_{i}=d_{i} x_{i}+\sum_{u \sim i} x_{u}$ (the eigenvalue equation for the signless Laplacian). Acting as in the proof of Theorem 3.1, if $x_{i}=1$ is the maximum absolute value of the coordinates of $\mathbf{x}$ and $j$ is a neighbour of $i$, we get

$$
\kappa_{1}\left(1+x_{j}\right)=\kappa_{1}+d_{j} x_{j}+\sum_{u \sim j} x_{u}
$$

which yields

$$
\kappa_{1}\left(1+x_{j}\right) \leq \kappa_{1}+2 d_{j}
$$

Taking the summation over all neighbours of $i$, we arrive at

$$
\kappa_{1}^{2} \leq \kappa_{1} d_{i}+2 d_{i} m_{i}
$$

Hence,

$$
\kappa_{1} \leq \frac{d_{i}+\sqrt{d_{i}^{2}+8 d_{i} m_{i}}}{2}
$$

along with equality if and only if $G$ is regular, which follows easily by considering the equality in (11). The latter inequality can be found in [5, p. 207], along with a different proof. By [3], for signed graphs with the same underlying graph, the maximum index is attained in the case of that with all edges being negative, up to a switching. In other words, every upper bound for $\kappa_{1}$ of $G$ is simultaneously an upper bound for $\mu_{1}$ of $\dot{G}=(G, \sigma)$. Thus, we have

$$
\mu_{1} \leq \frac{d_{i}+\sqrt{d_{i}^{2}+8 d_{i} m_{i}}}{2}
$$

Comparing the bounds (2) and (12), we easily obtain that the former one gives a better estimate whenever

$$
T_{i}^{+} \geq \frac{1}{8} d_{i}\left(3 d_{i}-\sqrt{d_{i}\left(d_{i}+8 m_{i}\right)}\right) .
$$

This, in particular, occurs whenever the right-hand side of the last inequality is negative.

We conclude by a lower bound for $\mu_{1}$. We say that a signed graph is net-regular if the difference between positive and the negative degree is constant on the vertex set. Such a signed graph does not need to be regular, but if it is, then we may write $r^{+}$and $r^{-}$for the positive and negative vertex degree, along with $r=r^{+}+r^{-}$. Observe that, in this case, $2 r^{-}$ appears as a Laplacian eigenvalue associated with a constant eigenvector.

Theorem 3.2. For the Laplacian index $\mu_{1}$ of a signed graph $\dot{G}$ with $n$ vertices,

$$
\mu_{1} \geq \frac{\sum_{i=1}^{n} d_{i}\left(d_{i}^{2}+\sum_{j \sim i} d_{j}-\sum_{j{ }_{j i}^{+}} d_{j}\right)}{\sum_{i=1}^{n} d_{i}^{2}},
$$

where $d_{i}$ denotes the degree of $i$.

Equality holds if $\dot{G}$ is switching equivalent to a regular and net-regular signed graph with $2 r^{-}$in the role of the Laplacian index.

Proof. For $L_{\dot{G}}=\left(l_{i, j}\right)$ and $\mathbf{d}=\left(d_{1}, d_{2}, \ldots, d_{n}\right)^{\top}$, using the Rayleigh principle, we get

$$
\mu_{1} \geq \frac{\mathbf{d}^{\top} L_{\dot{G}} \mathbf{d}}{\mathbf{d}^{\top} \mathbf{d}}=\frac{\sum_{i=1}^{n} \sum_{j=1}^{n} d_{i} l_{i, j} d_{j}}{\sum_{i=1}^{n} d_{i}^{2}}=\frac{\sum_{i=1}^{n}\left(d_{i}^{3}-\sum_{j \sim i} \sigma(i j) d_{i} d_{j}\right)}{\sum_{i=1}^{n} d_{i}^{2}},
$$

which leads to (13).

If $\dot{G}$ is switching equivalent to a regular (of degree $r$ ) and net-regular signed graph, then we have

$$
\mu_{1} \geq \frac{n r\left(r^{2}+r^{-} r-r^{+} r\right)}{n r^{2}}=r+r^{-}-r^{+}=2 r^{-} .
$$

By the assumption, we have $\mu_{1}=2 r^{-}$, and the result follows. 


\section{Acknowledgment}

The research of the second author is supported by the Serbian Ministry of Education, Science and Technological Development via the University of Belgrade.

\section{References}

[1] K. C. Das, An improved upper bound for Laplacian graph eigenvalues, Linear Algebra Appl. 368 (2003) $269-278$.

[2] J. S. Li, Y. L. Pan, De Caen's inequality and bounds on the largest Laplacian eigenvalue of a graph, Linear Algebra Appl. 328 (2001) 153-160.

[3] N. Reff, Spectral properties of complex unit gain graphs, Linear Algebra Appl. 436 (2012) 3165-3176.

[4] Z. Stanić, Bounding the largest eigenvalue of signed graphs, Linear Algebra Appl. 573 (2019) 80-89.

[5] Z. Stanić, Inequalities for Graph Eigenvalues, Cambridge University Press, Cambridge, 2015.

[6] Z. Stanić, Regular Graphs. A Spectral Approach, De Gruyter, Berlin, 2017.

[7] D. Stevanović, Spectral Radius of Graphs, Elsevier, Amsterdam, 2014.

[8] T. Zaslavsky, Matrices in the theory of signed simple graphs, In: B. D. Acharya, G. O. H. Katona, J. Nešetřil (Eds.), Advances in Discrete Mathematics and Applications: Mysore 2008, Ramanujan Math. Soc., Mysore, 2010, pp. 207-229. 